Изв. Крымской Астрофиз. Обс. 114, № 1, 43-48 (2018)

\begin{tabular}{c} 
ИЗВЕСТИЯ \\
КРЫМСКОЙ \\
АСТРОФИЗИЧЕСКОЙ \\
ОБСЕРВАТОРИИ \\
\hline
\end{tabular}

УДК 521.11, 521.182, 629.76

\title{
Динамическая эволюция космического мусора в окрестности областей движения спутников глобальных навигационных систем и геостационарной орбиты
}

\author{
Э.Д. Кузнещзов \\ Уральский федеральный университет, ул. Мира, 19, Екатеринбург, 620002 \\ eduard.kuznetsov@urfu.ru
}

Поступила в редакцию 15 ноября 2017 г.

\begin{abstract}
Аннотация. Рассмотрена динамическая эволюция космического мусора в окрестности активно используемых орбит: геостационарной и глобальных навигационных спутниковых систем ГЛОНАСС, GPS, Бэйдоу и Галилео, с целью оценить интервалы времени, необходимые для проникновения объектов космического мусора в область движения геостационарных и навигационных спутников. Начальные значения больших полуосей орбит выбирались на 500 км выше и ниже номинальных орбит навигационных спутников в окрестности резонансов высоких порядков, также были рассмотрены супергеосинхронные орбиты в окрестности резонансов высоких порядков. Отношение миделева сечения к массе варьировалось от малых значений, соответствующих спутникам, до больших значений, соответствующих фрагментам космического мусора. Орбитальная эволюция объектов моделировалась на интервале времени 240 лет с помощью "Численной модели движения искусственных спутников Земли”, разработанной в Томском государственном университете. Модель возмущающих сил учитывала: гравитационное поле Земли, притяжение Луны и Солнца, приливы в теле Земли, световое давление с учетом тени Земли, эффект Пойнтинга - Робертсона, сопротивление атмосферы. Уравнения движения интегрировались методом Эверхарта 19 порядка. Результаты существенно зависят от положения плоскости орбиты. Большие значения отношения миделева сечения $\mathrm{k}$ массе приводят к прохождению объектов через геостационарную область и область движения спутников глобальных навигационных систем вследствие светового давления и эффекта Лидова-Кодзаи, приводящих к долгопериодическим колебаниям эксцентриситета и наклона орбит.
\end{abstract}

DYNAMICAL EVOLUTION OF SPACE DEBRIS IN THE VICINITY OF REGIONS OF MOTION OF SATELLITES OF THE GLOBAL NAVIGATION SYSTEM AND THE GEOSTATIONARY ORBIT, by E.D. Kuznetsov. Dynamical evolution of space debris was considered in the vicinity of actively used orbits: geostationary and global navigation satellite systems GLONASS, GPS, BeiDou, Galileo. The aim of the research is to estimate time intervals which are necessary for space debris objects to get into the region of navigation satellites motion and the geostationary orbit. Initial orbits of objects were chosen $500 \mathrm{~km}$ above and below with respect to nominal semi-major axes of navigation orbits and on the resonant super-geostationary orbits. The area-to-mass ratio was varied from small values corresponding to satellites to high values which correspond to the space debris fragments. The orbital evolution of objects was modeled for 240 years using "Numerical Model of Motion of Artificial Satellites" elaborated at the Tomsk State University. The model of perturbing forces takes into account the major perturbing factors: gravitational field of the Earth, gravitation of the Moon and the Sun, tides of the Earth, direct radiation pressure by taking into consideration the Earth's shadow, the Poynting-Robertson effect, and 
the atmospheric drag. The equations of motion were integrated by Everhart's method of the $19^{\text {th }}$ order. Results significantly depend on the orbital plane position. High values of the area-to-mass ratio cause the objects to pass through the geostationary region and the region of navigation satellites motion due to the direct radiation pressure and the Lidov-Kozai effect, which produce long-period oscillations of eccentricity and orbit inclination.

Ключевые слова: динамическая эволюция, космический мусор, геостационарная орбита, глобальные навигационные спутниковые системы, ГЛОНАCC, GPS, Бэйдоу, Галилео, численное моделирование

\section{1 Введение}

Исследование долгопериодической эволюции объектов в окрестности геостационарной орбиты и орбит спутников глобальных навигационных систем становится все более актуальной задачей в связи с ростом числа объектов, запускаемых на эти орбиты. После завершения работы объекты должны переводиться на орбиты захоронения, хотя небольшая часть, включая аварийные аппараты, может оставаться в окрестности рабочих орбит. Под влиянием вековых лунно-солнечных резонансов наклоны и эксцентриситеты орбит испытывают долгопериодические возмущения большой амплитуды. Под действием этих возмущений объекты могут попадать в области движения активных спутников или входить в плотные слои атмосферы. Рост эксцентриситета и погружение перицентра орбиты в атмосферу Земли ведут к вековым возмущениям большой полуоси за счет диссипации энергии. Другой диссипативный эффект - эффект Пойнтинга - Робертсона - учитывается исключительно редко, хотя при больших и умеренных значениях парусности вековые возмущения большой полуоси могут приводить к существенному изменению орбитальной эволюции за счет прохождения через области резонансов.

В данной работе исследуются свойства орбит в окрестности резонансных областей с точки зрения использования этих орбит в качестве орбит захоронения.

Важным фактором, влияющим на динамическую эволюцию и обеспечивающим возможность объектам взаимодействовать с резонансами, является световое давление. Особенно сильно это проявляется при больших значениях парусности объектов.

Интерес к задаче о движении спутников с большой парусностью усилился после обнаружения в околоземном космическом пространстве более сотни объектов с парусностью от 1 до 50 м ${ }^{2} /$ кг (Фрю, Шилдкнехт, 2012). Парусность этих объектов, относимых к фрагментам космического мусора, значительно превышает значения, характерные для искусственных спутников Земли. При столь больших значениях парусности возмущения, обусловленные световым давлением, становятся вторым по величине, после гравитационного поля Земли, фактором, влияющим на движение тел в околоземном пространстве.

Световое давление оказывает существенное влияние на орбитальную эволюцию геосинхронных

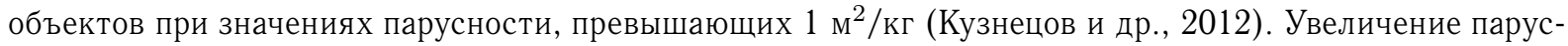
ности ведет к возрастанию амплитуд колебаний эксцентриситета и наклона орбиты и к сокращению периодов этих колебаний. Максимальные значения наклонов увеличиваются от $15^{\circ}$ (при парусности $1 \mathrm{~m}^{2} /$ кг) до $46^{\circ}$ (при парусности $30 \mathrm{~m}^{2} /$ кг) при сокращении периодов с 53 до 5 лет (Ансельмо, Пардини, 2005). При значениях парусности, превышающих $16 \mathrm{~m}^{2} /$ кг, в зависимости от начальных условий эксцентриситет орбиты может достигать значения 0.83 , обеспечивающего вход геостационарного объекта в плотные слои атмосферы Земли (Ансельмо, Пардини, 2005).

В работах (Чао, 2006; Валк и др., 2008) показано существование стационарной точки на фазовой плоскости “эксцентриситет - долгота перицентра”, обеспечивающей орбитальную эволюцию с почти постоянным средним значением эксцентриситета, если его начальное значение близко к 0.01 произведения парусности на коэффициент отражения поверхности спутника.

Исследование стохастических свойств движения геосинхронных объектов показало, что с ростом парусности увеличиваются и размеры зон стохастичности (Вытржыщак и др., 2007; Валк и др., 2009). 
Орбитальная эволюция объектов в окрестности резонанса 1:2 на длительных интервалах времени исследовалась в работах (Ансельмо, Пардини, 2009; Чао, Гик, 2004). Исследователи отмечают сложную зависимость результатов от начальных данных и от значений парусности.

Исследование орбитальной эволюции с большой парусностью на длительных интервалах времени требует учета влияния эффекта Пойнтинга - Робертсона. В статье (Кузнецов, 2011) выполнен анализ работ, посвященных учету влияния эффекта Пойнтинга-Робертсона и получены оценки вековых возмущений большой полуоси орбиты для геосинхронных спутников.

В работе (Алесси и др., 2014) рассмотрены стратегии по эффективному удалению космического мусора из областей движения спутников глобальных навигационных систем (GPS, Глонасс, Бэйдоу, Галилео).

В работе (Розенгрен и др., 2015) исследованы причины хаотической эволюции орбит навигационных спутников. Основным источником хаотической орбитальной эволюции являются вековые резонансы, связанные с движением узлов и перицентра лунной орбиты.

\section{2 Возможные орбиты захоронения для космического мусора}

В соответствии с рекомендациями Межагентского координационного комитета по космическому мусоpy (IADC, 2002), для объектов, завершивших работу на геостационарной орбите, определена большая полуось $a$ орбиты захоронения:

$$
a=a_{G E O}+\Delta a+1000 k \gamma
$$

Здесь $a_{G E O}$ - большая полуось геостационарной орбиты, $\Delta a=235$ км, $k$ - коэффициент отражения поверхности спутника, $\gamma$ - отношение миделева сечения к массе (парусность). Такой выбор большой полуоси орбиты захоронения обеспечивает защиту геостационарной области от объектов с малой парусностью, соответствующей парусности геостационарных спутников $\gamma=0.01-0.1 \mathrm{~m}^{2} /$ кг.

С течением времени объекты на орбитах захоронения могут разрушаться вследствие старения конструкции и материалов, столкновений, взрывов и др. Образующиеся фрагменты космического мусора, как правило, будут иметь парусность выше, чем парусность исходного объекта. Как показано в работах (Вытржыщак и др., 2007; Кузнецов, 2011), для объектов с большими значениями парусности $\gamma>1 \mathrm{~m}^{2} /$ кг орбита захоронения с большой полуосью, определяемой формулой (1), не обеспечивает безопасность геостационарной орбиты - фрагменты космического мусора проходят через геостационарную область. Поэтому в качестве возможных орбит захоронения геостационарных объектов были рассмотрены почти круговые (начальное значение эксцентриситета $e=0.001$ ) и почти экваториальные (начальное значение наклона $i=0.001^{\circ}$ ) супергеостационарные орбиты, расположенные в окрестности резонансов высоких порядков: 50:49 ( $a=42740$ км), 20:19 ( $a=43635$ км), 15:14 ( $a=44153$ км), 10:9 $(a=45237$ км $)$, 9:8 $(a=45613$ км $)$.

Таблица 1. Параметры орбит спутников глобальных навигационных систем и потенциальных орбит захоронения

\begin{tabular}{lccccc}
\hline Система & \multicolumn{2}{c}{ Номинальная орбита } & \multicolumn{2}{c}{ Орбита захоронения } & $i$, град \\
& Резонанс & $a$, км & Резонанс & $a_{d}$, км & \\
\hline ГЛОНАСС & $8: 17$ & 25508 & $14: 29$ & 25947 & 64.8 \\
GPS & $1: 2$ & 26559 & $15: 29$ & 27168 & 55 \\
Бэйдоу & $7: 13$ & 27907 & $4: 7$ & 29034 & 55 \\
Галилео & $10: 17$ & 29600 & $3: 5$ & 29994 & 56 \\
\hline
\end{tabular}

Для спутников глобальных навигационных систем, работающих на средних орбитах, политика увода отработавших спутников на орбиты захоронения еще не выработана. Орбиты спутников глобальных навигационных систем являются резонансными (табл. 1). Расстояние по большой полуоси между смежными системами составляет от 1000 до 1700 км, поэтому в качестве потенциальных орбит захоронения рассматривались орбиты в окрестности резонансов высоких порядков с большими полуосями $a_{d}$, превышающими соответствующие номинальные орбиты на 450-1100 км (табл. 1). 


\section{3 Численное моделирование}

Орбитальная эволюция объектов моделировалась на интервале времени 240 лет с помощью "Численной модели движения искусственных спутников Земли”, разработанной в Томском государственном университете. Модель возмущающих сил учитывает основные возмущающие факторы: гравитационное поле Земли (модель EGM96, гармоники до 27 порядка и степени включительно), притяжение Луны и Солнца, приливы в теле Земли, световое давление с учетом тени Земли (коэффициент отражения поверхности спутника $k=1.44)$, эффект Пойнтинга-Робертсона, сопротивление атмосферы. Уравнения движения интегрировались методом Эверхарта 19 порядка. Отношение миделева сечения к массе $\gamma$ варьировалось от малых значений, соответствующих спутникам, до больших значений, соответствующих фрагментам космического мусора.

Начальные значения долготы восходящего узла $\Omega$ полагались равными 0, 90, 180 и 270 градусов. Значение аргумента перицентра $g$ выбиралось из условия, чтобы в начальную эпоху при $\Omega=0^{\circ}$ перицентр орбиты был ориентирован на Солнце.

\section{4 Динамическая эволюция}

\section{1 Супергеостационарные орбиты}

Таблица 2. Интервалы времени $\Delta t$ (в годах) до первого проникновения фрагментов космического мусора в области движения активных спутников

\begin{tabular}{|c|c|c|c|c|c|}
\hline$\gamma, \mathrm{M}^{2} / \mathrm{K} \Gamma$ & ГЛОНАСС & GPS & Бэйдоу & Галилео & ГCO \\
\hline \multicolumn{6}{|c|}{ Резонанс 50:49 } \\
\hline 0.5 & - & - & - & - & 0.44 \\
\hline 9 & - & - & - & 33.73 & 0.03 \\
\hline 10 & - & - & 61.74 & 3.56 & 0.03 \\
\hline 11 & 167.72 & 88.62 & 7.53 & 0.41 & 0.03 \\
\hline \multicolumn{6}{|c|}{ Резонанс 20:19 } \\
\hline 1 & - & - & - & - & 161.23 \\
\hline 10 & - & - & - & 7.56 & 0.05 \\
\hline 11 & - & - & 46.49 & 0.47 & 0.05 \\
\hline 12 & - & 48.32 & 2.49 & 0.38 & 0.05 \\
\hline 13 & 19.36 & 2.52 & 0.41 & 0.33 & 0.03 \\
\hline \multicolumn{6}{|c|}{ Резонанс 15:14 } \\
\hline 2 & - & - & - & - & 0.27 \\
\hline 10 & - & - & - & 34.61 & 0.05 \\
\hline 12 & - & - & 3.50 & 0.38 & 0.05 \\
\hline 13 & 51.12 & 3.53 & 0.41 & 0.33 & 0.05 \\
\hline \multicolumn{6}{|c|}{ Резонанс 10:9 } \\
\hline 2 & - & - & - & - & 9.66 \\
\hline 10 & - & - & 190.69 & 69.57 & 0.08 \\
\hline 13 & - & 6.46 & 0.47 & 0.36 & 0.05 \\
\hline 14 & 4.49 & 0.49 & 0.38 & 0.30 & 0.05 \\
\hline \multicolumn{6}{|c|}{ Резонанс 9:8 } \\
\hline 4 & - & - & - & - & 0.22 \\
\hline 10 & - & - & 224.61 & 103.57 & 0.08 \\
\hline 13 & 211.33 & 7.47 & 0.49 & 0.36 & 0.08 \\
\hline
\end{tabular}

Для исследования динамической эволюции объектов на супергеостационарных орбитах рассматривались почти круговые (начальное значение $e=0.001$ ) и почти экваториальные (начальное значение $\left.i=0.001^{\circ}\right)$ орбиты, с начальными значениями больших полуосей в окрестности резонансов высоких порядков: 50:49 ( $a=42740$ км), 20:19 ( $a=43635$ км), 15:14 ( $a=44153$ км), 10:9 $(a=45237$ км), 
9:8 $\left(a=45613\right.$ км). Отношение миделева сечения к массе $\gamma$ варьировалось от $0.02 \mathrm{~m}^{2} /$ кг до максимально возможных значений, приводящих к падению на Землю.

Оценки интервалов времени $\Delta t$ до первого проникновения фрагментов космического мусора в области движения активных геостационарных и навигационных спутников существенно зависят от начального значения долготы восходящего узла $\Omega$. Наибольшие интервалы $\Delta t$ достигаются при начальном значении $\Omega=0^{\circ}$, что соответствует ориентации перицентра орбиты в направлении на Солнце. В табл. 2 приведены результаты для начального значения $\Omega=0^{\circ}$. Для каждой из областей даны минимальные значения отношения миделева сечения к массе $\gamma$, приводящие к проникновению, и соответствующие им интервалы $\Delta t$. Если для данного значения $\gamma$ объект проходит через несколько областей, то оценка $\Delta t$ приводится для каждой области.

\section{2 Орбиты в окрестности глобальных навигационных спутниковых систем}

Исследование динамической эволюции объектов на орбите, расположенной выше орбит спутников ГЛОНАСС в окрестности резонанса 14:29 с начальным значением большой полуоси $a=25947$ км и начальным значением наклона $i=64.8^{\circ}$, показывает, что объекты не попадают в области движения навигационных спутников, если начальное значение $\Omega=180^{\circ}$ и отношение миделева сечения к массе $\gamma<0.02 \mathrm{~m}^{2} /$ кг. Начальное значение $\Omega=0^{\circ}$ приводит к прохождению через области ГЛОНАСС, GPS, Бэйдоу и Галилео после $\Delta t=109,124,160$ и 181 года соответственно. Увеличение $\gamma$ ведет к сокращению интервалов времени $\Delta t$. При $\gamma=1 \mathrm{~m}^{2} /$ кг прохождения через области ГЛОНАCC, GPS, Бэйдоу и Галилео наступают при $\Delta t=0.4,26,75$ и 99 лет соответственно.

Анализ динамической эволюции объектов на орбите, расположенной выше орбит спутников GPS в окрестности резонанса 15:29 с начальным значениями $a=27168$ км и $i=55^{\circ}$, показывает, что объекты не попадают в области движения навигационных спутников при начальном значении $\Omega=0^{\circ}$ и $\gamma<0.2 \mathrm{~m}^{2} /$ кг. При начальном значении $\Omega=180^{\circ}$ и $\gamma=0.02 \mathrm{~m}^{2} /$ кг прохождения через области ГЛОНАСС, GPS, Бэйдоу и Галилео наступают после $\Delta t=194,159,163$ и 216 лет соответственно, а при $\gamma=1 \mathrm{~m}^{2} /$ кг интервалы времени сокращаются до $\Delta t=75,0.4,21$ и 98 лет соответственно.

Рассмотрение динамической эволюции объектов на орбите, расположенной выше орбит спутников Бэйдоу в окрестности резонанса $4: 7$ с начальными значениями $a=29034$ км и $i=55^{\circ}$, показывает, что объекты не попадают в области движения навигационных спутников, если начальное значение $\Omega=0^{\circ}$ или $180^{\circ}$ и $\gamma<0.2 \mathrm{~m}^{2} /$ кг. Начальное значение $\Omega=90^{\circ}$ при $\gamma=0.02 \mathrm{~m}^{2} /$ кг приводит к прохождению через области Бэйдоу и Галилео после $\Delta t=221$ и 190 лет соответственно. Начальное значение $\Omega=270^{\circ}$ при $\gamma=0.02 \mathrm{~m}^{2} /$ кг ведет к прохождению через области ГЛОНАСС, GPS, Бэйдоу и Галилео спустя $\Delta t=195,173,140$ и 110 лет соответственно.

Исследование динамической эволюции объектов на орбите, расположенной выше орбит спутников Галилео в окрестности резонанса $3: 5$ с начальными значениями $a=29994$ км и $i=56^{\circ}$, показывает, что объекты испытывают существенные лунно-солнечные возмущения и возвращаются в область системы Галилео спустя $\Delta t=99,170,201$ и 85 лет для начальных значений $\Omega=0^{\circ}, 90^{\circ}, 180^{\circ}$ и $270^{\circ}$, соответственно, при $\gamma=0.02 \mathrm{~m}^{2} /$ кг.

\section{5 Заключение}

В результате проведенного исследования установлено, что динамическая эволюция объектов на орбитах захоронения существенно зависит от начального положения плоскости орбиты и парусности $\gamma$ объектов. Большие значения отношения миделева сечения к массе $\gamma$ приводят к прохождению объектов через геостационарную область и область движения спутников глобальных навигационных систем вследствие светового давления и эффекта Лидова-Кодзаи, приводящих к долгопериодическим колебаниям эксцентриситета и наклона орбит.

Работа выполнена при поддержке постановления № 211 Правительства Российской Федерации (контракт № 02.А03.21.0006) и Министерства образования и науки Российской Федерации (базовая часть государственного задания, РК № AАAА-A17-117030310283-7). 


\section{Литература}

Алесси и др. (Alessi E.M., Rossi A., Valsecchi G.B., Anselmo L., Pardini C., et al.) // Acta Astron. 2014. V. 99. P. 292.

Ансельмо, Пардини (Anselmo L., Pardini C.) // 4th European Conf. on Space Debris. / Ed. Danesy D. Noordwijk: ESA Publications Division. 2005. P. 279.

Ансельмо, Пардини (Anselmo L., Pardini C.) // Adv. Space Res. 2009. V. 43. P. 1491.

Валк и др. (Valk S., Lemaitre A., Anselmo L.) // Adv. Space Res. 2008. V. 41. P. 1077.

Валк и др. (Valk S., Delsate N., Lemaitre A., Carletti T.) // Adv. Space Res. 2009. V. 43. P. 1509.

Вытржыщак и др. (Wytrzyszczak I., Breiter S., Borczyk W.) // Adv. Space Res. 2007. V. 40. P. 134.

Кузнецов Э.Д. // Астрон. вестник. 2011. Т. 45. С. 444.

Кузнецов Э.Д., Захарова П.Е., Гламазда Д.В., Шагабутдинов А.И., Кудрявцев С.О. // Астрон. вестник. 2012. Т. 46. С. 480.

Розенгрен и др. (Rosengren A.J., Alessi E.M., Rossi A., Valsecchi G.B.) // Mon. Not. Roy. Astron. Soc. 2015. V. 449. P. 3522.

Фрю, Шилдкнехт (Fruh C., Schildknecht T.) // Mon. Not. Roy. Astron. Soc. 2012. V. 419. P. 3521.

Чao (Chao C.C.) // AIAA/AAS Astrodynamics Specialist Conf. Keystone, Colorado. 2006. No. AIAA2006-6514. 8 p.

Чао, Гик (Chao C.C., Gick R.A.) // Adv. Space Res. 2004. V. 34. P. 1221.

Inter-Agency Space Debris Coordination Committee (IADC): IADC Space Debris Mitigation Guidelines, IADC-02-01, October 15, 2002. 